\title{
Dieulafoy's disease: endoscopic treatment and follow up
}

\author{
B Baettig, W Haecki, F Lammer, R Jost
}

\begin{abstract}
The findings from 480 patients who had emergency endoscopy for acute upper gastrointestinal bleeding of non-variceal origin at our institution were analysed. Twenty eight patients $(5.8 \%)$ had a Dieulafoy lesion. In 27 patients $(96.4 \%)$ bleeding could be successfully managed by injection of norepinephrine and polidocanol, in repeated sessions if needed. Two patients had to be treated surgically: one because of uncontrollable bleeding from the Dieulafoy lesion and one despite endoscopic control of the bleeding Dieulafoy lesion because of a concomitant bleeding from an anastomosal ulcer after gastric resection. Three patients died during hospital stay from causes unrelated to bleeding from Dieulafoy lesion. Out of the 25 patients discharged from the hospital 21 treated by endoscopy and two treated with surgery were followed up for a mean of 28.3 and 22.5 months, respectively. Twenty endoscopically treated patients $(95 \%)$ had no recurrence of Dieulafoy's bleeding. One patient experienced severe rebleeding from the original site after a transient endoscopy confirmed complete disappearance. He had emergency operation without a further attempt to control bleeding by endoscopy. It is concluded that bleeding from Dieulafoy's disease can be successfully managed by endoscopic injection treatment. The longterm outcome is favourable.
\end{abstract}

Department of Internal Medicine, and

B Baettig

F Lammer

Gastroenterology

Division, University

Hospital of Zurich

Switzerland

W Haecki

R Jost

Correspondence to:

Dr R Jost, Gastroenterology

Division, University Hospita

of Zurich, Rämistrasse 100 8091 Zurich, Switzerland.

Accepted for publication 26 January 1993
(Gut 1993; 34: 1418-1421)

Dieulafoy's disease is a well known entity characterised by a bleeding or clot bearing artery protruding into the intestinal lumen without surrounding ulceration. It may occur anywhere in the gastrointestinal tract but is predominantly located in the gastro-oesophageal junction and in the stomach along the lesser curvature. ${ }^{1-3}$ The Figure shows the typical histological picture of a Dieulafoy lesion. Aneurysmas, atherosclerosis or vasculitis are generally absent. ${ }^{13-8}$

The incidence of Dieulafoy's lesions among

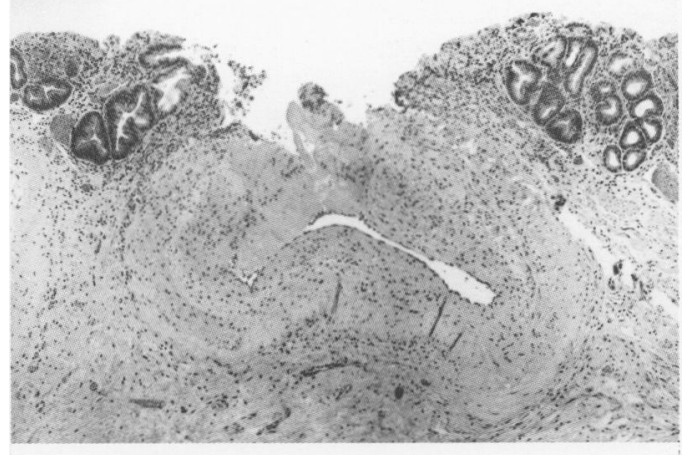

patients with acute upper gastrointestinal bleeding varies from $0.5 \%$ to $14 \%$, mainly depending upon selection criteria. There is a general consensus that the disease is 'underdiagnosed rather than truly rare' as the lesion may be very discrete and needs a high degree of suspicion to be detected. ${ }^{1-39-11}$

The disease used to be fatal frequently ${ }^{12-1+}$ because of the non-visualisation of the vessel and 'blind' gastric resection with recurrent haemorrhage postoperatively. By endoscopy the nature of the bleeding site can be identified and the operation planned accordingly. Furthermore, the possibility of endoscopic intervention adds a new perspective to the management of Dieulafoy's disease. The definitive treatment is still controversial. Some advocate primary endoscopic intervention, ${ }^{12}{ }^{15-19}$ reserving the surgical option for failures. Others prefer primary surgical intervention, assuming a high risk of recurrence, and see primary endoscopic treatment as an alternative in patients with a high operative risk only. ${ }^{35102021}$

The aim of our study was to assess the efficacy and longterm effectiveness of treatment with endoscopy in Dieulafoy's disease.

\section{Materials and methods}

From January 1984 to October 1991, 480 patients with acute upper gastrointestinal bleeding of non-variceal origin were admitted to our gastroenterology department. After basic life support all patients had emergency endoscopy. Twenty eight patients $(5 \cdot 8 \%)$ had Dieulafoy's disease. In all patients an attempt was made to treat the lesion by injection of up to $10 \mathrm{ml}$ epinephrine $(1: 10000)$ and up to $6 \mathrm{ml} 1 \%$ polidocanol. The injection was usually performed at different sites around the vessel and directly into the vessel. Adherent clots had to be removed in nine cases to prove the nature of the lesion. To achieve optimal diagnostic accuracy all initial diagnostic and interventional endoscopies were done or supervised by very experienced members of the staff and virtually all patients were re-examined after the initial interventional endoscopy. Where there was rebleeding, endoscopic treatment was repeated. If repeated endoscopic attempts failed, emergency operation was performed. All patients were treated with $\mathrm{H} 2$ receptor antagonists after the procedure.

Each patient was evaluated as to sex, age, past medical history, use of drugs, alcohol abuse, haemoglobin values at entry, blood replacement, additional endoscopic findings, bleeding type, localisation of the lesion, and the final outcome. Follow up data could be obtained from 23 of the 25 patients discharged from the hospital either significant surrounding inflammation (Case No 4 haematoxilin and eosin original magnification $\times 40$ ). 
TABLE I Details of the patients

\begin{tabular}{|c|c|c|c|c|c|}
\hline $\begin{array}{l}\text { Case } \\
\text { no }\end{array}$ & Age & Sex & Medical history & $\begin{array}{l}\text { Alcohol } \\
\text { abuse }\end{array}$ & Treatment \\
\hline 1 & 73 & M & Septicaemia, death due to septicaemia & No & NSAIDs \\
\hline 2 & 59 & M & Four days after operation of a plexus papilloma & No & None \\
\hline 3 & 44 & $\mathbf{M}$ & History of gastric resection and anastomosal bleeding & Yes & None \\
\hline 4 & 25 & M & None & No & None \\
\hline 5 & 70 & $\mathbf{F}$ & $\begin{array}{l}\text { One week after endoscopic papillotomy for } \\
\text { choledocholithotomy }\end{array}$ & No & None \\
\hline 6 & 62 & M & Alcoholic liver cirrhosis & Yes & None \\
\hline 7 & 47 & M & Alcoholic liver disease & Yes & None \\
\hline 8 & 59 & $\mathrm{~F}$ & History of gastric resection & No & None \\
\hline 9 & 63 & M & Erysipela & No & NSAIDs \\
\hline 10 & 71 & M & $\begin{array}{l}\text { History of fundoplication, recurrent gastric and duodenum } \\
\text { ulcers }\end{array}$ & No & None \\
\hline 11 & 45 & M & Coronary artery disease & Yes & None \\
\hline 12 & 32 & $\mathrm{~F}$ & One week after renal transplantation & No & None \\
\hline 13 & 70 & M & Coronary artery disease & No & NSAIDs \\
\hline 14 & 50 & M & Coronary artery disease & No & NSAIDs \\
\hline 15 & 90 & $\mathrm{~F}$ & Coronary artery disease & No & None \\
\hline 16 & 69 & M & Alcoholic liver cirrhosis & Yes & None \\
\hline 17 & 75 & M & Coronary artery disease & No & Salicylates \\
\hline 18 & 46 & $\mathbf{M}$ & None & Yes & None \\
\hline 19 & 70 & M & Pulmonary embolism, death due to cardiac failure & No & None \\
\hline 20 & 78 & M & Coronary artery disease & No & NSAIDs \\
\hline 21 & 72 & $\mathbf{F}$ & Pneumonia, death due to multiorgan failure & No & NSAIDs \\
\hline 22 & 68 & M & Cerebral stroke & No & None \\
\hline 23 & 60 & M & Renal insufficiency & No & Salicylates \\
\hline 24 & 71 & $\mathbf{F}$ & Polyarthrosis & No & NSAIDs \\
\hline 25 & 66 & M & Oesophageal varices, alcoholic liver cirrhosis & Yes & NSAIDs \\
\hline 26 & 82 & $\mathrm{M}$ & Coronary artery disease, cerebral stroke & No & Salicylates \\
\hline 27 & 65 & M & Polymyalgia rheumatica & No & NSAIDs \\
\hline 28 & 45 & $\mathbf{M}$ & History of gastric resection & Yes & None \\
\hline
\end{tabular}

NSAID $=$ non-steroidal anti-inflammatory drugs.

directly from the patients or by interviewing their physicians.

Standard statistical methods were performed using Student's unpaired $t$ test and $\chi^{2}$ analysis.

\section{Results}

AGE AND SEX

The Dieulafoy group consisted of 22 men $(78.6 \%)$ and six women $(21 \cdot 4 \%)$. The mean age (SD) was $61 \cdot 7$ years (25-90 years (15.043)). There was no statistical age difference between the Dieulafoy group and patients with bleeding ulcers of the stomach $(n=100 ; 61.7 v 63.3$ years, $\mathrm{p}=0.67)$. The sex distribution, however, showed statistically more male patients in the Dieulafoy $\operatorname{group}(p=0.04)$.

\section{MEDICAL HISTORY, TREATMENT/DRUGS BEFORE ADMISSION}

Table I lists the age and sex distribution, medical history, alcohol consumption, and concomitant treatment of the patient population.

CLINICAL PRESENTATION AND BLEEDING HISTORY In 21 patients Dieulafoy's lesions presented with sudden and massive gastric bleeding with haematemesis and melaena. Seven patients suffered from melaena alone. On admission the mean haemoglobin value was $9.2 \mathrm{mg} \%$

TABLE II Site of the Dieulafoy's lesions

\begin{tabular}{ll}
\hline $\begin{array}{l}\text { Stomach }(\mathrm{n}=24) \\
\text { Within } 6 \mathrm{~cm} \text { from gastro-oesophageal junction }\end{array}$ & - towards the lesser curve $(\mathrm{n}=8)$ \\
$\quad(\mathrm{n}=17)$ & - towards the major curve $(\mathrm{n}=2)$ \\
& - towards the anterior wall $(\mathrm{n}=3)$ \\
& - under the Z-line in a hiatus hernia $(\mathrm{n}=2)$ \\
Corpus $(\mathrm{n}=5)$ & \\
Proximal the anastomosis after gastric resection $(\mathrm{n}=2)$ & \\
Duodenum $(\mathrm{n}=4)$ & \\
Duodenal bulb $(\mathrm{n}=3)$ & \\
Duodenal knee $(\mathrm{n}=1)$ & \\
\hline
\end{tabular}

$(+/-2 \cdot 16 \mathrm{~g} \%)$. There was a mean requirement of 6.6 units of blood $(5 \cdot 1 \mathrm{U})$. Patients with concomitant alcoholic hepatopathy/cirrhosis presented with a mean haemoglobin value of $10 \cdot 1 \mathrm{~g} \%(+/-1 \cdot 5 \mathrm{~g} \%)$.

In 23 cases, no previous bleeding episodes were known. Three patients had a history of previous bleeding from ulcers of the duodenum or stomach, one patient experienced anastomosal bleeding with a history of gastric resection, one patient had a Dieulafoy's lesion treated conservatively in another hospital with recurrent bleeding episodes within one year before admission.

ENDOSCOPIC FINDINGS AND TREATMENT

In 24 patients $(86 \%)$ the Dieulafoy disease was located within the stomach; in 17 patients $(61 \%)$ it was found at the classic site within $6 \mathrm{~cm}$ from the gastro-oesophageal junction (in eight cases near the lesser curve, in two near the greater curve, in three on the anterior wall, in two on the posterior wall, and in two in a hiatus hernia immediately below the oesophageal-gastric junction); in five patients in the corpus; and in two patients proximal from the anastomosis after gastric resection. Four further bleeding sites (14\%) were detected in the duodenum (see Table II).

All patients were treated endoscopically by injection treatment (one with combined use of heater probe), whether they were actively bleeding or not. Nineteen patients $(68 \%)$ were actively bleeding during emergency endoscopy. In 15 cases endoscopic treatment was successful after one treatment session, in two cases after the second, and in one case after the third. One patient had to have emergency operation because of uncontrollable bleeding. None of the nine patients with an adherent clot at first examination developed major rebleeding.

Overall, endoscopic treatment was successful in 27 patients $(96 \%)$ during the initial hospital period. One patient had elective surgical revision despite bleeding control for a concomitant bleeding anastomosal ulcer after gastric resection. Another patient experienced a recurrence of Dieulafoy's lesion half a year after an initially successful endoscopic approach and had surgical intervention without a further endoscopic attempt (histologically proved case of Dieulafoy's disease, see the Figure). Interestingly, a control endoscopy six weeks after the initial successful injection treatment showed a healed mucosal defect with no visible vessel left.

Other endoscopic findings included one case with anastomosal bleeding (history of gastric resection), three cases with oesophageal varices, one of which was combined with fundic varices (all without signs of recent haemorrhage), four cases with oesophagitis, two cases with nonbleeding duodenal (one with combined gastric ulcers), and two cases with gastric ulcers.

Endoscopic diagnosis of Dieulafoy's lesion was made in 23 cases $(82 \%)$ in the first, in four $(14 \%)$ in the second, and in one case $(4 \%)$ in the third endoscopic session. The main reason for repeated endoscopies was poor visualisation because of large amounts of blood in the stomach or wide areas of adherent haematin. Concomi- 
tant treatment consisted of $\mathrm{H} 2$ antagonists in all cases.

PATHOLOGICAL EXAMINATION

Pathological examination of the resected specimens in two cases showed the typical appearance of Dieulafoy's lesion. In one further case with concomitant gastric ulcer bleeding the specimen was not checked histologically for Dieulafoy's disease.

\section{COMPLICATIONS AND DEATH}

Three patients died during the hospital stay because of diseases unrelated to Dieulafoy's disease (pulmonary embolism, septicaemia, and pneumonia with multiorgan failure). One patient experienced a bleeding episode associated with a treatment induced ulcer 10 days after the initial treatment, which was successfully treated with another injection. None of the other patients had serious complications otherwise.

\section{LATE CLINICAL RESULTS}

Of the 25 patients discharged either after surgical (two cases) and endoscopic ( 23 cases) treatment, 23 could be followed up either by direct contact or by information obtained from their physicians. Twenty two patients have hitherto not suffered from recurrence of Dieulafoy's bleeding. As mentioned above, one patient experienced severe rebleeding six months after initial successful sclerotherapy and has had no rebleeding after emergency operation until now (four months). Out of these, 10 patients died of causes unrelated to Dieulafoy lesion bleeding with a mean follow up of 13.8 months (minimum: 2 months, maximum 54 months). Of the four patients with alcoholic hepatopathy/hepatic cirrhosis, one has recovered (follow up: 41 months) and three have died (two in hepatic coma, the other with lethal oesophageal variceal bleeding; mean follow up five months). The follow up periods ranged from 2 to 80 months with a mean of $28 \cdot 3$ months in the endoscopically $(n=21)$ and 22.5 in the surgically $(n=2)$ treated group.

\section{Discussion}

With the advent of diagnostic and therapeutic endoscopy the management of Dieulafoy's disease has been directed towards a more careful surgical intervention on the one hand and successful bleeding control by injection treatment on the other hand. ${ }^{121516}$ In daily practice as well as in the recent studies Dieulafoy lesion is diagnosed most often by endoscopy. . $^{1-35-19}$

Although not randomised and with a small number of histopathologically confirmed cases, we believe, that the large number of patients in our study successfully managed by injection treatment alone favours a primary endoscopic approach towards Dieulafoy's disease. It must be emphasised, however, that an experienced endoscopist is necessary, as Dieulafoy's disease can easily be overlooked or concomitant lesions, such as ulcers or varices, may wrongly be considered responsible for the bleeding episode. Our diagnostic efficiency is shown by a diagnostic yield in the first endoscopic session of $82 \%$; further sessions were needed in the rest of patients mainly because of non-removable blood clots. Three patients had repeated unsuccessful diagnostic endoscopies or angiographies in referring hospitals. We therefore believe, that in unclear cases of severe upper gastrointestinal bleeding a vigorous attempt should be made to identify the lesion to avoid unnecessary surgery. Furthermore close study is mandatory to detect rebleeding after treatment.

With regard to the differential diagnosis to clot covered lesions mimicking Dieulafoy lesion and fundic varices, we strictly applied the diagnosis to a vessel without surrounding ulceration after removal of the clot. No Dieulafoy lesion was found in the fundus and anaemia was not so pronounced in the patients with alcoholic hepatopathy/cirrhosis to lead to a confusion with bleeding fundic varices. In our patients, no endoscopic findings suggestive of stress ulcers were seen.

In $61 \%$ the lesion was found at the classic site within $6 \mathrm{~cm}$ of the gastro-oesophageal junction, mostly near the lesser curve. Similar to other reports ${ }^{41522}$ we also identified Dieulafoy's lesion in the duodenum.

The effectiveness of the endoscopic treatment is supported by our high incidence of bleeding arrest in $95 \%$ of actively bleeding lesions. In this group three cases had repeated but eventually successful therapeutic endoscopies. There were no rebleeding episodes in the group which were not actively bleeding after the first treatment. In the series of Pointner ${ }^{1}$ there seems to be fewer episodes of rebleeding using a combined regimen of injection treatment and electrocoagulation than with either procedure alone. The optimal regimen is not clearly established and should be studied in a randomised trial. Our treatment success rate lies within other authors' experience with local endoscopic treatment, reporting success rates ranging from 82 (1) to $100 \%,{ }^{219}$ with much shorter study periods, however.

Interestingly, one patient experienced recurrence of Dieulafoy's lesion at the identical site half a year after successful treatment and with healing proved endoscopically. Because of this case and because of the studies of the support$\mathrm{ers}^{5}{ }^{10}$ of primary surgical intervention an objection, until now, was the rather short follow up periods of the larger series. ${ }^{12}$ Our study with a mean follow up period of 28.3 months (range 2-80) clearly shows the longterm effectiveness of endoscopic treatment of Dieulafoy's lesion.

Dieulafoy's lesion is a disease of mainly middle aged and elderly male patients. Our results with a mean age of 61.7 and a more pronounced male predominance of nearly $1: 5$ are in agreement with former findings. ${ }^{279}$

As a result of restricting our study to nonvariceal bleeding and a high referral rate from other hospitals there is a seemingly high incidence of Dieulafoy's lesion in our patients. Therefore, our data cannot show the true incidence.

Clinically, 21 patients presented with sudden and massive haematemesis and melaena and seven patients with melaena alone. The severity 
of the bleeding is manifested by the number of blood units required (average 6.6 units, minimum 2; maximum 26 units).

The pathogenesis of Dieulafoy's disease is still unclear. There is a calibre persistency of some arteries instead of a regular reduction in size before the vessels penetrate the layers of the intestinal wall. ${ }^{823-25}$ Disagreement exists with respect to the further pathogenetic steps that lead to rupture of the vessel. Perhaps additional factors such as alcoholism, chronic gastritis, and chronic use of drugs are responsible in some cases. ${ }^{5726}$ In our series alcohol abuse is found in $25 \%$, salicylates and non-steroid anti-inflammatory drug use in $39 \%$ and concurrent peptic disease in $11 \%$. As there are no control groups described so far, the causes of the disease remain speculative. Interestingly, there was no statistical age difference between patients diagnosed as having Dieulafoy's type of bleeding and patients with diagnosed bleeding ulcers of the stomach. On the other hand, there was a significantly higher proportion of men among patients with Dieulafoy's disease than among those with bleeding ulcers of the stomach. Whether the same average onset of gastric ulcer and Dieulafoy's type of bleeding is merely coincidential or suggests some common pathophysiological cause remains to be answered. The fact that in three cases concomitant gastric ulcers were found should not discourage the endoscopist to look for further possible bleeding sites despite a seemingly obvious source of bleeding. While some authors have reported a frequent history of peptic ulcer disease, ${ }^{47927}$ data on concomitant peptic ulcer disease are lacking, mainly because in most studies this question was not studied specifically.

The medical histories of our patients show a high incidence of concomitant or past diseases with only two patients having an absolutely unremarkable history. Some authors have also described significant concurrent morbidity or remarkable past medical history, ${ }^{346910171828}$ while others have described the 'typical patient' with Dieulafoy's lesion to have an unremarkable past medical history. ${ }^{121}$ On the basis of our experience and the conflicting findings reported we feel a 'typical patient' syndrome for Dieulafoy's disease remains speculative.

In summary, we conclude that endoscopic injection treatment is safe and effective and is the first choice in the management of Dieulafoy's disease with excellent longterm results. Surgery should only be considered if primary endoscopic treatment fails.

We thank Dr $\mathbf{P}$ Vogt for providing the Figure of the resected specimen and Professor R Ammann and Professor H E Blum for specimen and Professor R Ammann
their helpful comments and support.
1 Pointner R, Schwab G, Königsrainer A, Dietze O. Endoscopic treatment of Dieulafoy's disease. Gastroenterology 1988; 94: 563-6.

2 Nicolet M, Cuttat J-F, Dorta G, Fried M, Blum AL Exulceratio simplex Dieulafoy de l'estomac: à propos de cas. Schweiz Med Wochenschr 1990; 120: 1421-4.

3 Bakka A, Rosseland AR. Massive gastric bleeding from Exulceratio simplex (Dieulafoy). Acta Chir Scand 1986; 152 285-8.

4 Durham JD, Kumpe DA, Rothbarth LJ, Van Stiegman G. Dieulafoy disease: Arteriographic findings and treatment. Radiology 1990; 174: 937-41.

5 Goossens A, Pipeleers-Marchial M, Peeters O, Delvaux G. Solitary exulceratio simplex (Ulcer of Dieulafoy). Acta Chir Belg 1989; 89: 54-7.

6 Schwesinger G, Scülfort H. Exulceratio simplex Dieulafoy. $Z$ Gesamte Inn Med 1987; 42: 654-5.

7 Juler GL, Labitzke HG, Lamb R, Allen R. The pathogenesis of Dieulafoy's gastric erosion. Am $\mathcal{F}$ Gastroenterol 1984; 79: of Dieulafos.

8 Miko TL, Thomazy VA. The caliber persistent artery of the stomach: a unifying approach to gastric aneurysm, Dieulafoy's lesion, and submucosal arterial malformation Hum Pathol 1988; 19: 914-21.

9 Peitsch W, Lange W, Schauer A. Die Exulceratio simplex Dieulafoy. Dtsch Med Wochenschr 1987; 112: 1940-2.

10 Scully RE, Mark EJ, McNeely BU, McNeely BU. Case records of the Massachusetts General Hospital. N Engl f Med 1991; 325: 1086-96.

11 Umlauft M, Zimmermann G. Die Exulceratio simplex Dieulafoy. Wien Klin Wochenschr 1977; 89: 404-6.

12 Dieulafoy G. Exulceratio simplex: L'intervention chirurgicale dans les hematemeses foudroyantes consecutives à dans les hematemeses foudroyantes consecutives à
l'exulceration simple de l'estomac. Bull Acad Med 1898; 39: 49-84

13 Demling L, Elster K, Frick W, Gall F, Ottenjann R. Der kranke Magen. München, Berlin: Urban \& Schwarzenberg, 1970

14 Wanke M. Spezielle pathologische Anatomie. In: Doer W, Seifert G, Uehlinger E, eds. Berlin: Springer Verlag, 1971 $330-4$.

15 Goldenberg St P, DeLuca VA, Marignani P. Endoscopic treatment of Dieulafoy's lesion of the duodenum. $A m \mathcal{F}$ Gastroenterol 1990; 85: 452-4.

16 Boron B, Mobarhan S. Endoscopic treatment of Dieulafoy hemorrhage. $\mathcal{F}$ Clin Gastroenterol 1987; 9: 518-20.

17 Helliwell M, Irving JD. Haemorrhage from gastric artery aneurysms. $B M F$ 1981; 282: 460-

18 De Jaureguiberry JP, Moreau X, Duval JL, Theobald X, Quino JF. Slérothérapie endoscopique d'une ulcération de Dieulafoy de siège duodénal. La Presse Médicale 1991; 20 128.

19 Hwai-Jeng L, Fa-Yauh L, Yang-Te T, Shou-Dong L, Chen-Hsen L, Wei-Ming K. Therapeutic endoscopy for Dieulafoy's disease. F Clin Gastroenterol 1989; 11 : 507-10.

20 Richards WO, Grove D, Williams LF. Hemorrhage from a Dieulafoy type ulcer of the colon: A new cause of lower gastrointestinal bleeding. Am Surg 1988; 54: $121-4$.

21 Velhuyzen van Zanten SJO, Bartelsman JFWM, Schipper MEI, Tytgat GNJ. Recurrent massive haematemesis from MEI, Tytgat GNJ. Recurrent massive haematemesis rom Gieulafoy vascular malfo

22 McClave SA, Goldschmid S, Cunningham JT, Boyd WP Dieulafoy's cirsoid aneurism of the duodenum. Dig Dis $\mathrm{Sci}$ 1988; 33: 801-5.

23 Voth D. Das architektonische Prinzip der Magenarterien in seiner Bedeutung für die Magenblutung. Zentralbl Allg Pathol 1962; 103: 553-4

24 Eidus LB, Rasculi P, Manion D, Heringer R. Caliber persistent artery of the stomach (Dieulafoy's vascular malformation). Gastroenterology 1990; 99: 1507-10.

25 Molnar P, Miko T. Multiple arterial caliber persistence resulting in haematomas and total rupture of the gastric wall. Am 7 Surg Pathol 1982; 6: 83-6.

26 Schmid KW, Pointner R, Feichtinger J, Schmid KW Exulceration simplex Dieulafoy of the colon - A case report Endoscopy 1988; 20: 88-9.

27 Saueracker AJ, Van Stiegmann G, Pearlman NW. Dieulafoy's Disease: A not uncommon clinical dilemma. Dig Surg 1988; 5: 89-94.

28 Mortensen NJ, Mountford RA, Davies JD, Jeans WD. Dieulafoy's disease: a distinctive arteriovenous malformation causing massive gastric haemorrhage. $\mathrm{Br}$ f Surg 1983 ; 70: 76-8. 